\title{
O Papel do Profissional do Design no Aprimoramento de um Ambiente Virtual de Aprendizagem em Linguagem Infantil
}

The role of the design professional in the improvement of a virtual environment of learning in children's language El papel del profesional del diseño en la mejora de un entorno virtual de aprendizaje en el lenguaje de los niños

\author{
Líliam Maria Dib MARSON ${ }^{1}$ \\ Camila de Castro CORRÊA ${ }^{2}$ \\ Maria Gabriela CAVALHEIRO ${ }^{3}$ \\ Aline MARTINS ${ }^{4}$ \\ Luciana Paula MAXIMINO5
}

${ }^{1}$ Mestre em ciências, Departamento de Fonoaudiologia, Faculdade de Odontologia de Bauru, USP, Bauru - SP, Brasil ${ }^{2}$ Docente da Universidade de Brasília e do Centro Universitário Planalto do Distrito Federal, Doutora em Bases Gerais da Cirurgia, Faculdade de Medicina de Botucatu, UNESP, Botucatu/SP, Brasil

${ }^{3}$ Doutora em ciências da reabilitação, Hospital de Reabilitação em Anomalias Craniofaciais, USP, Bauru - SP, Brasil

${ }^{4}$ Mestre em ciências, Departamento de Fonoaudiologia, Faculdade de Odontologia de Bauru, USP, Bauru - SP, Brasil

${ }^{5}$ Professora Associada do Departamento de Fonoaudiologia, Faculdade de Odontologia de Bauru, USP, Bauru - SP, Brasil

\section{Resumo}

Objetivo: adaptar e analisar o ambiente virtual de aprendizagem "Fonoaudiologia para Pediatras" sobre a aquisição e desenvolvimento da linguagem infantil, realizando um re-design para melhorar a qualidade gráfica e a interação digital. Métodos: Participaram do estudo 115 profissionais das áreas do design gráfico, web design e publicidade. A avaliação do material foi realizada por meio de um questionário de caracterização e do instrumento WebMAC - Website Motivational Analysis Checklist for Evaluating Service-Based Commercial Web Sites. Resultados: Foram levantados os problemas de desing do ambiente virtual de aprendizagem e realizadas as alterações no re-desing quanto a identidade visual, conjunto visual, nome, endereço eletrônico e menu, mantendo o seu subnome e conteúdo na íntegra. A fim de melhorar a navegabilidade e acesso das informações, foi realizada a assinatura da plataforma Wordpress e para melhor memorização, o registro do domino foi alterado para "Fonopediatria". O conteúdo foi classificado como "Curso Impressionante", e o Website como Excelente. Conclusão: Desta forma, o ambiente virtual de aprendizagem foi disponibilizado em: http://www.fonopediatria.com.br, e o processo de avaliação por designers foi efetivo para conferir a qualidade do Ambiente Virtual de Aprendizagem, considerando o ambiente excelente, com alta expectativa para o sucesso.

Descritores: Educação à Distância; Telemedicina; Tecnologia Educacional.

Abstract

Aims: to adapt and to analyze the Virtual Learning Environment "Fonoaudiologia para Pediatras" regarding the acquisition and development of the language in children, performing a re-design of the environment to improve their graphic quality and their digital interaction. Methods: 115 professionals from the areas of graphic design, web design and advertising participated in the study. The evaluation of the material was carried out by means of a pre-questionnaire (characterization) and the WebMAC instrument - Website Motivational Analysis Checklist for Evaluating Service-Based Commercial WebSites. Results: The design problems were raised to adapt and made changes in the re-design as to the visual identity, visual set, name, e-mail address and menu, keeping its subname and content in full. In order to improve the navigability and access of the information was carried out the signature of the platform Wordpress and for better memorization, the registration of the domino was changed to "Fonopediatria". Content has been rated as "Awesome Course," and the Website is Excellent. Conclusion: In this way, the Virtual Learning Environment was made available at: http://www.fonopediatria.com.br and the evaluation process by designers was effective to check the quality of the Virtual Learning Environment, considering the excellent environment, with high expectations for the success.

Descriptors: Education, Distance; Telemedicine; Educational Technology.

\section{Resumen}

Objetivos: Adaptar y analizar el Entorno Virtual de Aprendizaje "Fonoaudiologia para Pediatras" en cuanto a la adquisición y desarrollo del lenguaje en los niños, realizando un rediseño del entorno para mejorar su calidad gráfica y su interacción digital. Métodos: participaron en el estudio 115 profesionales de las áreas de diseño gráfico, diseño web y publicidad. La evaluación del material se llevó a cabo mediante un pre-cuestionario (caracterización) y el instrumento WebMAC - Website Motivational Analysis Checklist para la Evaluación de Sitios Web Comerciales Basados en Servicios. Resultados: Se plantearon los problemas de diseño para adaptar y realizar cambios en el rediseño en cuanto a la identidad visual, conjunto visual, nombre, dirección de correo electrónico y menú, manteniendo su subnombre y contenido completo. Con el fin de mejorar la navegabilidad y acceso de la información se realizó la firma de la plataforma Wordpress y para una mejor memorización, se cambió el registro del dominó a "Fonopediatria". El contenido ha sido calificado como "Curso impresionante" y el sitio web es excelente. Conclusión: De esta manera, se puso a disposición el Ambiente Virtual de Aprendizaje en: http://www.fonopediatria.com.br y el proceso de evaluación por parte de los diseñadores fue efectivo para verificar la calidad del Ambiente Virtual de Aprendizaje, considerando el excelente ambiente, con altas expectativas de éxito.

Descriptores: Educación a Distancia;Telemedicina; Tecnología Educacional.

INTRODUÇÃO

O Design tem grande potencial para participar e colaborar com a educação formal e informal por meio do uso dos seus fundamentos, metodologias de trabalho, maneira de interagir na formação da cultura material, de proceder na concepção dos objetos, de utilizar as tecnologias e os materiais do seu característico sentido estético enquanto atividade projetual, de realizar a leitura e a configuração do que está ao redor ${ }^{1,2}$.
O Design Gráfico tem função de conceber, programar, projetar e realizar comunicações visuais destinadas a transmitir mensagens específicas a grupos determinados. É possível visualizar as demais áreas sob o enfoque do Design pela sua capacidade e necessidade de participar e elaborar projetos colaborativos e interdisciplinares com diferentes profissionais $^{3-5}$. 
Sendo assim, o designer é responsável pela comunicação e pelo sucesso da repercussão de um produto, um projeto ou uma empresa, trabalha em diversas áreas, tem uma atuação ampla e busca a inclusão de todas as camadas da sociedade, sem preconceitos, valorizando sempre a interdisciplinaridade. Diante desta interdisciplinaridade, a atuação conjunta com outras áreas é de grande relevância, especialmente na execução de ações conjuntas com objetivos comuns ${ }^{2,5}$.

Em 2011, foi realizado um estudo pioneiro integrando a área do Design Gráfico com a Fonoaudiologia, desenvolvendo 0 Ambiente Virtual de Aprendizagem (AVA) "Olhar além do Som" para introduzir Português e Libras para crianças deficientes auditivas da pré-escola até o ensino fundamental de maneira mais atrativa e lúdica, o qual demonstrou contribuir para aprendizagem ${ }^{6}$.

A união das Ciências Fonoaudiologia e Design é um desafio, especialmente para a realização de projetos em Teleducação, como por exemplo, na elaboração de AVAs. Para isto, é fundamental criar novos acessos à informação, gerar maior interação do profissional com o conhecimento por meio de professores capacitados, por meio de recursos modernos de computação e materiais didáticos atrativos/funcionais ${ }^{2,5}$.

Os blogs, exemplos de Ambiente Virtual de Aprendizagem (AVA), deixaram de ser unicamente "diários" on-line para assumir funções mais significativas nos processos sociais, pelo dinamismo na difusão de ideias, possibilidade de interação e facilidade de atualização das informações ${ }^{7-9}$. Ressalta-se a necessidade de se realizar um briefing antes da criação destes AVAs, contemplando as seguintes etapas: coleta de dados, análise/definição do público-alvo, do tema, os objetivos educacionais, os conteúdos a serem abordados e a infra-estrutura tecnológica a ser utilizada para o desenvolvimento, implementação e manutenção do $\operatorname{AVA}^{10}$.

Alguns AVAs já foram desenvolvidos para facilitar a comunicação entre fonoaudiólogos, especialistas e não especialistas em determinada área da Fonoaudiologia, como é o caso do website voltado para a formação de fonoaudiólogos a respeito da intervenção de fala em indivíduos com Fissura Labiopalatina e outras anomalias craniofaciais $^{11}$. Sobre a temática da linguagem infantil em AVAs, houve um trabalho voltado para a população de pais e cuidadores de bebês $^{12}$ e outro voltado para a orientação de profissionais $^{13}$.
Em vista desta demanda de desenvolver trabalhos interdisciplinares para melhorar a qualidade dos serviços de saúde oferecidos e realizar prevenção, diagnóstico e intervenção da linguagem infantil, foi construído um AVA sobre aquisição e desenvolvimento da linguagem infantil ${ }^{13}$, "Fonoaudiologia para Pediatras", para orientação de médicos pediatras. Este material foi avaliado do ponto de vista da qualidade técnica e do conteúdo por 63 fonoaudiólogos, sendo avaliado como "Excelente", além de alcançar 5.046 visitas de diversos países, demonstrando o uso frequente da Internet para obtenção de informações na área da saúde.

Desta forma, o objetivo da presente pesquisa foi realizar um re-design no AVA "Fonoaudiologia para Pediatras" para melhorar sua qualidade gráfica e sua interação digital, buscando, além da interação entre a Medicina e a Fonoaudiologia, agregar o trabalho da área de Design, otimizando o aproveitamento da informação sobre linguagem infantil para os profissionais.

MATERIAL E MÉTODO

- Aspectos Éticos

O presente estudo foi aprovado pelo Comitê de Ética e Pesquisa em Seres Humanos da instituição envolvida, sob $\mathrm{n}^{\circ}$ de protocolo 18472813.7.0000.5417.

- Casuística

Foram convidados a participar do estudo, profissionais de diferentes áreas do Design. Como estratégia para alcançar o maior número de profissionais de diferentes regiões, foi enviada uma carta convite via e-mail para os membros cadastrados nos grupos Design Unesp, Design Faal e Behance da rede social Facebook. O convite descrito continha, sucintamente, os objetivos do trabalho e o link do AVA, que direcionava os interessados em participar com link direto para o ambiente.

Participaram do estudo 115 profissionais das áreas do Design, os quais avaliaram a qualidade gráfica e funcional do material e dos recursos tecnológicos, em outras palavras, avaliaram o layout, a estrutura de navegação, a identidade visual e as imagens.

Aprendizagem do Ambiente Virtual de

O AVA foi desenvolvido por Martins ${ }^{13} \mathrm{e}$ disponibilizado inicialmente na web no link: $<$ http://fonoaudiologiaparapediatras.wordpress.c om>, que também pode ser referido como blog, dividido em 11 seções independentes quanto a disponibilização do conteúdo (Figura 1).

Primeiramente, foi realizado um levantamento de todos os problemas gráficos 
identificados, quanto a identidade visual, conjunto visual, nome, endereço eletrônico e menu, e desenvolvido um briefing de todas as alterações necessárias para adaptação do AVA.

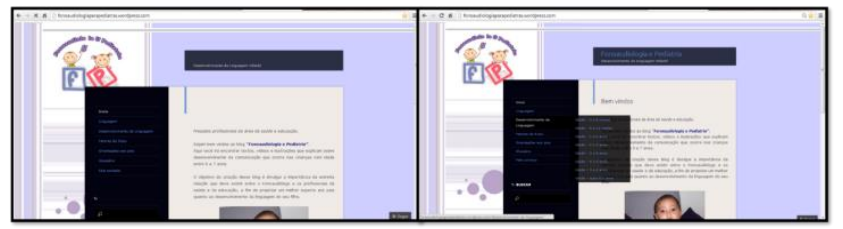

Figura 1: Tela inicial do AVA "Fonoaudiologia para pediatras", com demonstração do menu.

\section{- Instrumentos de avaliação}

A avaliação do material foi realizada por meio de dois questionários. A avaliação inicial constava de um pré-questionário, no qual 0 usuário não se identificava, mas fornecia informações básicas referentes a sua área de atuação em Design, ano de formação e cidade em que atua.

Em seguida, aplicou 0 instrumento WebMAC - Website Motivational Analysis Checklist for Evaluating Service-Based Commercial Web Sites para checar se o layout, a estrutura de navegação, a identidade visual e as imagens estariam adequados ao assunto, ao público e ao objetivo do $\mathrm{AVA}^{14}$. É composto por 32 enunciados, pontuados da seguinte maneira: (3) concordo completamente, (2) concordo parcialmente, (1) discordo parcialmente, (0) discordo completamente. Os enunciados são agrupados em 4 domínios: "ESTIMULANTE", "SIGNIFICATIVO", "ORGANIZADO" E "FÁCIL DE USAR"

Após realizada a pontuação de cada domínio individualmente, eles são agrupados conforme a expressão: $V=E+S$; $X S=O+F$. A pontuação $V$ é a soma dos domínios "ESTIMULANTE" e "SIGNIFICATIVO" e reflete a dimensão Valor, ou seja, o quanto é valioso este programa de capacitação. A pontuação XS é a soma dos domínios "ORGANIZADO" e "FÁCIL DE USAR" e reflete a dimensão Expectativa para o Sucesso, que são por fim, projetados em um plano cartesiano. Se os pontos, ou uma grande quantidade dos pontos individuais se localizarem dentro do segundo quadrante, significa que o website se enquadra em "Curso Impressionante", avaliando-o positivamente.

Para a inserção dos questionários no AVA, foi utilizado o Google DOCs que permite a elaboração de questionários e planilhas on-line. Os dados dos usuários e dos questionários foram mantidos em sigilo e foram acessados apenas pelos pesquisadores.

RESULTADOS

○ Re-Design do Ambiente Virtual de
Aprendizagem

De acordo com o levantamento dos problemas e da elaboração do briefing, foram realizadas as seguintes alterações:

Quadro 1. Alterações realizadas no re-design do AVA quando à identidade visual, conjunto visual, nome, endereço eletrônico e menu.

\begin{tabular}{|c|c|c|}
\hline CATEGORIA & $\begin{array}{c}\text { PROBLEMAS } \\
\text { IDENTIFICADOS }\end{array}$ & $\begin{array}{l}\text { ALTERAÇÕES } \\
\text { REALIZADAS }\end{array}$ \\
\hline $\begin{array}{l}\text { Identidade } \\
\text { Visual }\end{array}$ & $\begin{array}{l}\text { Não deixa claro qual é o } \\
\text { nome do AVA, pois o título } \\
\text { é "Fonoaudiologia e } \\
\text { Pediatria", reforçado pela } \\
\text { identidade visual. Já o } \\
\text { endereço eletrônico } \\
\text { denomina-se } \\
\text { "Fonoaudiologia para } \\
\text { pediatras", deixando uma } \\
\text { dúvida de qual } \\
\text { nomenclatura deve ser } \\
\text { utilizada para referenciar o } \\
\text { conteúdo. } \\
\text { A compreensão do nome do } \\
\text { AVA na identidade visual } \\
\text { não é rápida e fácil, pois } \\
\text { apresenta um corte na } \\
\text { palavra "Fonoaudiologia" } \\
\text { além de estar com um } \\
\text { tamanho de fonte pequeno, } \\
\text { dificultando a leitura e } \\
\text { compreensão da identidade } \\
\text { visual. }\end{array}$ & $\begin{array}{l}\text { Foi modificada a nomenclatura } \\
\text { tanto para o endereço } \\
\text { eletrônico quanto para o título } \\
\text { do AVA e identidade visual. } \\
\text { Foi elaborado um logotipo de } \\
\text { fácil leitura e memorização, } \\
\text { destacando a nomenclatura } \\
\text { para melhor compreensão do } \\
\text { nome do AVA e da identidade } \\
\text { visual e foi elaborado um } \\
\text { símbolo de fácil compreensão } \\
\text { referenciando a a a } \\
\text { Fonoaudiologia com o público } \\
\text { principal, no caso, os Pediatras. }\end{array}$ \\
\hline Conjunto Visual & $\begin{array}{l}\text { A tela de corpo de texto do } \\
\text { AVA cobre parte da } \\
\text { identidade visual, } \\
\text { principalmente com o rolar } \\
\text { da página, cobrindo o } \\
\text { logotipo pela metade. } \\
\text { O título do AVA e subtítulo } \\
\text { estão em tamanho de fonte } \\
\text { pequeno dificultando a } \\
\text { leitura e compreensão pelo } \\
\text { usuário. } \\
\text { O espaço total do conjunto } \\
\text { visual (tela de fundo e tela } \\
\text { de corpo do AVA) não está } \\
\text { bem distribuído deixando } \\
\text { um grande espaço vazio do } \\
\text { lado direito da tela. }\end{array}$ & $\begin{array}{l}\text { A tela de corpo de texto do AVA } \\
\text { foi modificada, permitindo } \\
\text { live visibilidade e maior } \\
\text { destaque da identidade visual. } \\
\text { O título do AVA e subtítulo } \\
\text { foram dispostos com maior } \\
\text { destaque, para facilitar a } \\
\text { leitura e compreensão pelo } \\
\text { usuário. } \\
\text { O espaço total do conjunto } \\
\text { visual (tela de fundo e tela de } \\
\text { corpo), foi organizado de } \\
\text { maneira proporcional, sem } \\
\text { espaços vazios ou muito cheios } \\
\text { para facilitar a leitura e } \\
\text { usabilidade. }\end{array}$ \\
\hline Nome & 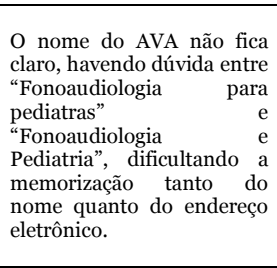 & $\begin{array}{l}\text { O nome do AVA foi alterado, } \\
\text { deixando clara a proposta do } \\
\text { material, mantendo-se o } \\
\text { mesmo, tanto para o título do } \\
\text { AVA quanto para o endereço } \\
\text { eletrônico, sem deixar dúvidas } \\
\text { entre qual nomenclatura deve } \\
\text { ser utilizada para referenciar o } \\
\text { ambiente e permitindo uma } \\
\text { melhor memorização pelo } \\
\text { usuário. }\end{array}$ \\
\hline $\begin{array}{l}\text { Endereço } \\
\text { eletrônico }\end{array}$ & $\begin{array}{l}\text { O endereço eletrônico do } \\
\text { AVA é extenso, dificultando } \\
\text { a memorização e acesso } \\
\text { pelo usuário. }\end{array}$ & 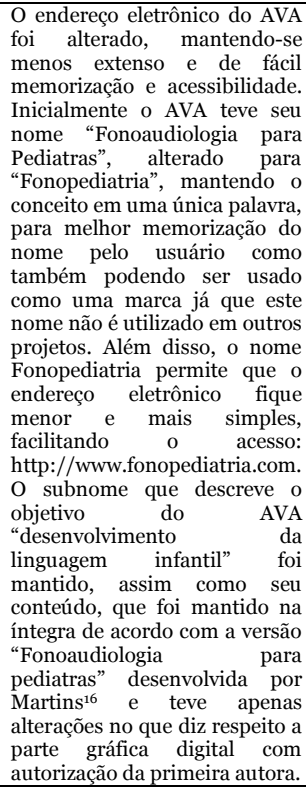 \\
\hline Menu & $\begin{array}{l}\text { O menu de conteúdos do } \\
\text { AVA está na mesma barra } \\
\text { lateral do que outros links } \\
\text { sugeridos pelo ambiente } \\
\text { confundindo a } \\
\text { navegabilidade do usuário } \\
\text { com muita informação } \\
\text { além de estar com tamanho } \\
\text { de fonte pequena, } \\
\text { dificultando a visibilidade e } \\
\text { leitura. }\end{array}$ & $\begin{array}{l}\text { O menu foi alterado, mantido } \\
\text { em destaque separado dos } \\
\text { outros elementos informativos } \\
\text { do AVA para melhor } \\
\text { usabilidade do usuário, } \\
\text { permitindo que ele encontre as } \\
\text { informações das quais precisa } \\
\text { com maior facilidade e rapidez. }\end{array}$ \\
\hline
\end{tabular}


O AVA teve o layout e a identidade visual produzidos com os recursos do Design Gráfico, utilizando-se da plataforma do Wordpress (na versão custeada para possibilitar a utilização de mais recursos gráficos) e dos softwares Adobe Photoshop e Corel Draw. Além disso, outros elementos oferecidos pelo AVA foram agregados para melhor navegabilidade e acesso a informações, como contador mundial, que disponibiliza a localidade e quantidade de acessos dos usuários, a barra de busca, e pesquisa e links úteis relacionados ao tema. Para isso, foi realizada a assinatura da plataforma Wordpress e o registro do domínio do AVA "Fonopediatria", permitindo o acesso ao AVA pelo endereço eletrônico http://www.fonopediatria.com.br e a construção da nova versão do AVA mantendo o seu conteúdo na íntegra.

A produção da nova identidade visual foi elaborada pelo software Corel Draw versão X5 utilizando o novo nome "Fonopediatria" e empregando signos relacionados à Fonoaudiologia e ao mundo infantil (Figura 3). A imagem do logotipo remete a um balão de fala, construindo por meia face de uma criança do sexo feminino e meia face de uma criança do sexo masculino, com as cores azul e rosa representando os sexos. $O$ nome "Fonopediatria" tem a nomenclatura "Fono" em destaque, pois o foco principal do projeto é a Fonoaudiologia. Foram utilizadas as seguintes famílias tipográficas para a elaboração da identidade visual: Today-SHOP Light e TodaySHOP Medium.

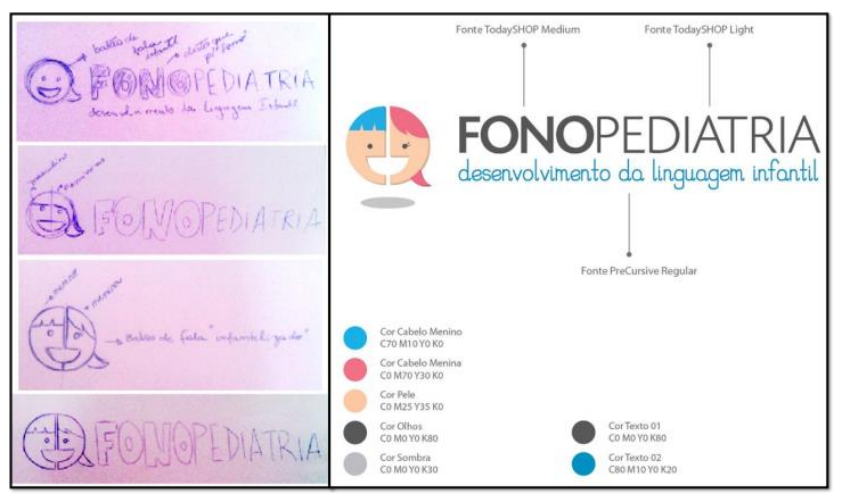

Figura 2: Croquis do Logotipo da versão "Fonopediatria" e versão final do logotipo da versão "Fonopediatria".

A foto de capa e o fundo do AVA foram compostos por um mosaico de fotos de sete crianças, demonstrando seu desenvolvimento cronológico, disposto no cabeçalho do ambiente. Todas as fotos são de crianças familiares das autoras do AVA e foram autorizadas para uso.

O menu do AVA foi disposto separadamente da barra lateral vertical, em uma barra horizontal bem no início da página, abaixo do logotipo, permitindo que o menu ficasse mais organizado e separado dos links e dos questionários avaliativos. Os conteúdos foram mantidos na íntegra alterando apenas a disposição dos elementos no menu.

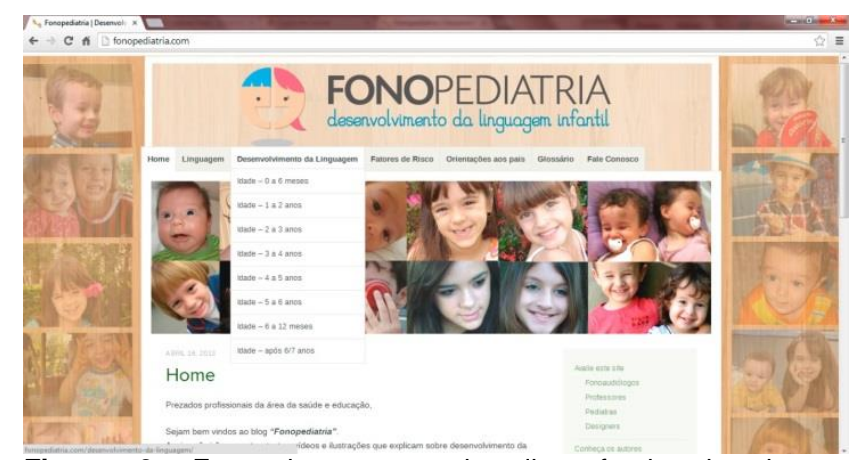

Figura 3: Foto de capa, cabeçalho, fundo, logotipo e demonstração do menu em barra horizontal do AVA "Fonopediatria".

A barra lateral foi disposta do lado direito, contendo os questionários avaliativos, a bibliografia e as informações sobre os autores, tendo logo abaixo uma lacuna para pesquisa, seguida dos links relacionados com o projeto: ASHA, FOB-USP, HRAC, Portal dos Bebês e Telessaúde Brasil. No final, mais abaixo, encontra-se uma imagem com os órgãos patrocinadores e o número de acessos ao ambiente.

Instrumentos de avaliação

O AVA "Fonopediatria" ficou disponibilizado oito meses (período do trabalho de re-design e avaliação) recebendo 3.188 visitas, tendo uma média de 75 visitas por dia. As localidades das visitas também foram registradas, observando que várias visualizações foram realizadas em outros países, além do Brasil, como Japão, Estados Unidos, Portugal, Canadá, Irlanda, Argentina, Colombia, México, Espanha, Índia, Romênia e Bolívia

Os participantes designers eram dos seguintes estados: São Paulo, Brasília, Santa Catarina, Mato Grosso e Mato Grosso do Sul. Com uma prevalência de atuação em Design Gráfico (Tabela 1).

Tabela 1. Número total de participantes por área específica do Design.

\begin{tabular}{lc}
\hline \multicolumn{1}{c}{ Área de atuação em Design } & Total \\
\hline Design gráfico & 61 \\
Design de produto & 20 \\
Design de comunicação & 6 \\
Design de interação & 7 \\
Design tipográfico & 2 \\
Design estratégico & 2 \\
Design visual & 2 \\
Design de jogos & 1 \\
Design editorial & 1 \\
Design de serviços & 2 \\
Design de mobiliário & 4 \\
Outra área do Design & 7 \\
\hline \multicolumn{2}{c}{ SOMA } \\
\multicolumn{2}{c}{} \\
\hline \multicolumn{2}{c}{}
\end{tabular}




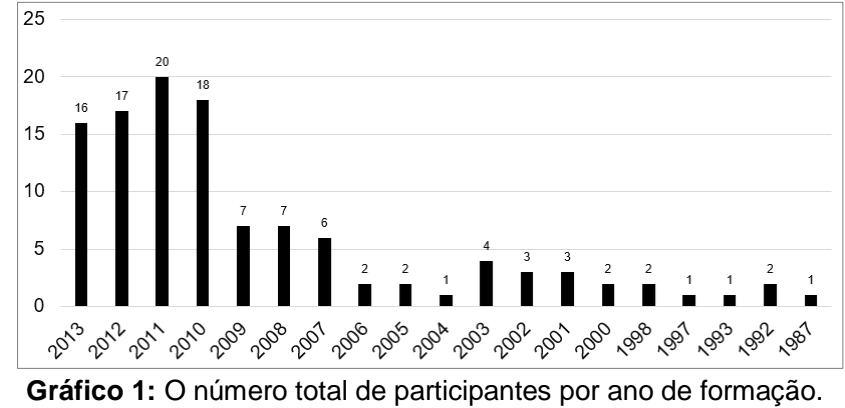

Em relação às respostas do questionário WebMAC, observou a distribuição de respostas conforme a Tabela 2, resultando na projeção cartesiana do Gráfico 2, demonstrando a expectativa de sucesso e dimensão Valor do AVA, que correspondeu à classificação de "Curso Impressionante", avaliando-o positivamente, como website Excelente.

Tabela 2. Número de respostas para cada questão do questionário WebMAC.

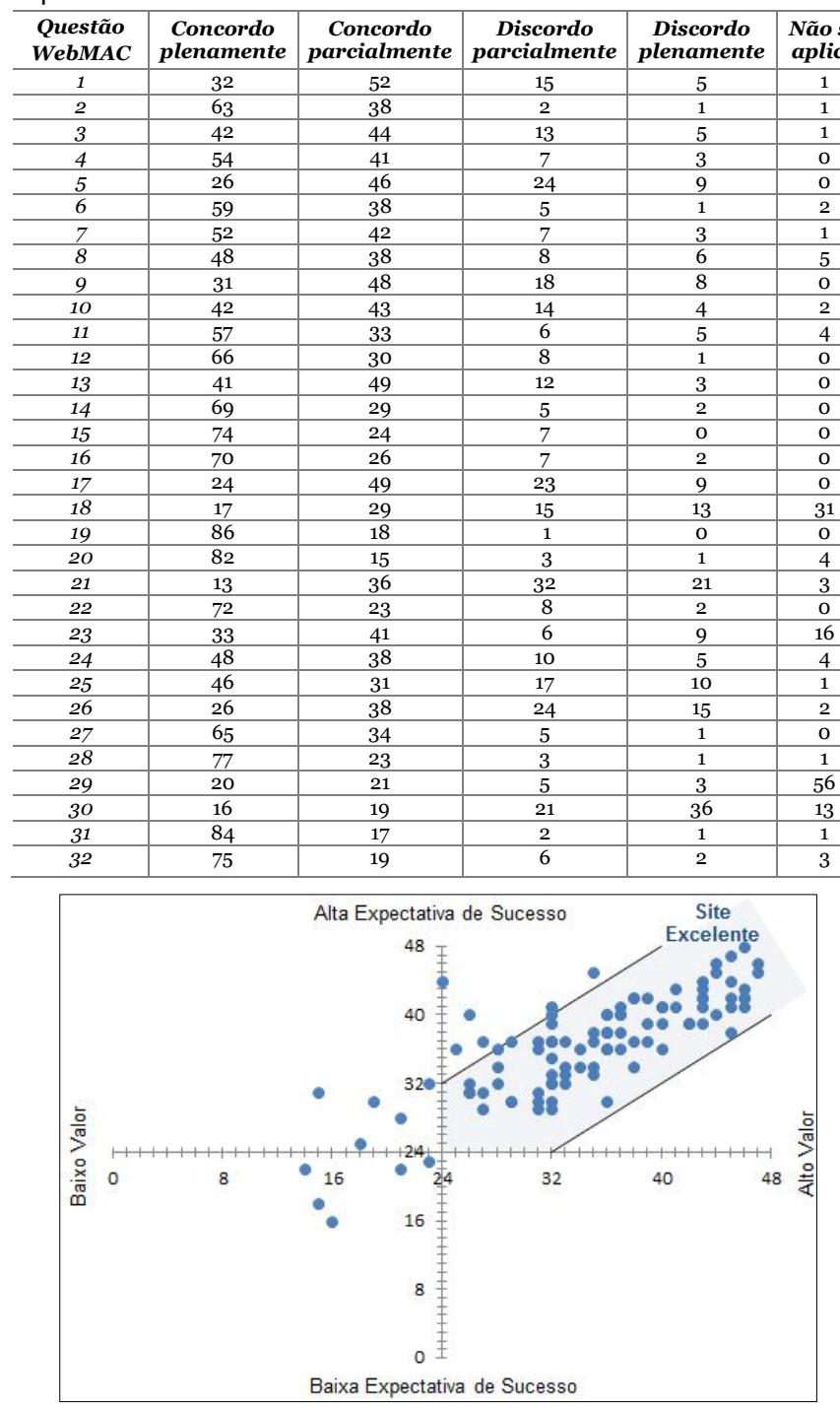

Gráfico 2. Demonstração cartesiana do Valor e Expectativa do AVA "Fonopediatria".

Para que o AVA possa ser melhorado futuramente e se tornar ainda mais adequado para o uso pelos usuários, os participantes responderam duas questões discursivas.
1) O que foi de maior motivação no AVA:

- Fornecer informação

- Conscientizar

- Orientações sobre linguagem infantil

- Logotipo no topo da página

- Foto de capa

- Fotos das crianças

- Fácil de usar

- Visual condizente com o tema

2) Sugestões:

- O Logotipo direcionar o usuário para a home page ao clicar

- Melhorar corpo de texto (tamanho da fonte)

- O AVA apresenta "cara de blog". Um site, segundo sugestões, daria mais credibilidade.

- Tirar as fotos do fundo.

DISCUSSÃO

Este estudo englobou o re-design gráfico digital do AVA "Fonoaudiologia para pediatras" bem como a análise da qualidade destas modificações na nova versão proposta, intitulada "Fonopediatria". Manteve-se o conteúdo original da primeira versão, resultado de um trabalho interdisciplinar entre as áreas de Design Gráfico e Fonoaudiologia, agregando o uso de tecnologias para disponibilizar informações a respeito do desenvolvimento de linguagem e garantir um ambiente atrativo e agradável ao público alvo.

O relato da atuação interdisciplinar entre as ciências envolvidas denota o caráter original do estudo em questão. A participação integrada do profissional do Design no desenvolvimento de sistemas midiáticos e hipermidiáticos aplicados a EaD é indispensável, uma vez que este profissional tem habilidades para configurar transformando a informação possibilitando a melhor integração homem-máquina ou homeminformação $0^{1-3,15}$.

O AVA criado por Martins ${ }^{13}$ teve como propósito atingir a população de médicos pediatras com a finalidade de orientá-los quanto ao desenvolvimento infantil no âmbito da teleducação interativa. No entanto, o material não foi avaliado do ponto de vista da qualidade gráfica. Os resultados obtidos revelaram que o redesign efetuado foi avaliado como excelente, com alta expectativa para 0 sucesso. A utilização do blog para a transmissão de conhecimento deve ser valorizada devido à flexibilidade, interatividade, cooperação no processo da aprendizagem construtivista, democratizando o acesso à informação ${ }^{9}$.

A adequação ou re-design do AVA englobou a identificação dos problemas a execução propriamente dita. A análise das mudanças necessárias e elaboração do briefing 
para que fosse possível desenvolver um material prático e de qualidade, que estimulasse a navegação e usabilidade do AVA, foi a fase que demandou mais tempo. Todo esse processo se fundamenta na complexidade do processo educativo, que abrange inclusive aspectos individuais motivacionais, em que a área do design tem a contribuir com ênfase no que se aos blogs ${ }^{10}$.

Houve a preocupação em desenvolver um ambiente acessível e organizado, visualmente agradável e de fácil utilização, por meio das ferramentas do Design Gráfico e da plataforma escolhida (Wordpress).

$\mathrm{Na}$ fase re-Design preocupou-se principalmente com a disposição dos elementos em um layout adequado e uma identidade visual limpa, que abrangesse o tema, que fosse de fácil leitura e memorização e com o título e endereço eletrônico menos extenso. Além disso, houve o cuidado em elaborar um ambiente o mais usual possível não somente para o público alvo, ou seja, pediatras, mas também aos demais profissionais da área da saúde, educadores e pais. Verificou-se que as pessoas retêm mais as informações quando estas são transmitidas de maneira clara e principalmente com o acompanhamento de imagens e ferramentas adequadas que contribuem para a memorização, interatividade e usabilidade, por meio de um conjunto composto de uma identidade visual, elementos gráficos visuais de fundo e estrutura gráfica visual $^{7}$.

Durante 8 meses em que o AVA esteve disponível na web houve intenso acesso. De acordo com as informações de acessos registrados, observou-se atividade de usuários de outros países, não só do Brasil. Este achado confirma a aplicabilidade em diferentes contextos com ampla abrangência ultrapassando as barreiras geográficas como denota os estudos em EaD ${ }^{16}$.

Ressalta-se que não houve dificuldade quanto ao recrutamento dos avaliadores designers, em sua predominância da área do design gráfico, seguido do design de produto, nos últimos 4 anos de formação e do estado de São Paulo. Pode-se inferir, que o predomínio das participações de profissionais do estado de São Paulo, deve-se a influência da Universidade de origem da pesquisadora que mediou sua rede de contatos, que foi uma das formas de acesso e busca do público de participantes.

Considerando a análise descritiva dos resultados obtidos por meio das porcentagens gerais, para os itens "concordo plenamente", "concordo parcialmente", "discordo parcialmente", "discordo plenamente" e "não se aplica", foi observada maior pontuação no item "concordo parcialmente" e "discordo parcialmente" para a maioria das questões.

Evidencia-se que essas questões quando analisadas utilizando os critérios e a fórmula proposta pelo questionário WebMAC, obtiveram pontuação considerada excelente. Logo, como resultado da avaliação final do AVA realizada por designers, englobando a qualidade geral e a qualidade gráfica digital, o atributo deste foi caracterizado como excelente, ou seja, este AVA é uma fonte excelente de informação da saúde, os usuários poderão acessar, navegar e interagir facilmente no ambiente e com as informações contidas nele. Neste mesmo sentido, profissionais médicos receberam conteúdo por meio de um AVA sobre medicina nuclear, o qual também foi avaliado e considerado relevante, viabilizando troca de experiências com profissionais de diferentes regiões do Brasil $^{17}$. Além disto, esta característica da interatividade ressaltada no questionário WebMAC permite com que haja a estimulação do raciocínio clínico-epidemiológico na área da medicina, especificamente ${ }^{18 .}$

Considerando os resultados obtidos neste estudo como um AVA de excelente qualidade, a etapa seguinte do trabalho consistirá em aumentar a divulgação da nova versão do AVA para propiciar difusão de informações de saúde, bem como projetos interdisciplinares com profissionais de outras áreas que possam contribuir para o crescimento e desenvolvimento do AVA.

CONCLUSÃO

Este trabalho desenvolveu um novo layout e uma nova identidade visual para o AVA desenvolvido por Martins ${ }^{16}$, bem como modificou o nome do AVA intitulado de "Fonoaudiologia para Pediatras" para "Fonopediatria". O AVA "Fonopediatria" encontra-se disponível para acesso na internet, pelo endereço eletrônico: http://www.fonopediatria.com.br.

O processo de avaliação por designers foi efetivo para avaliar a qualidade do AVA, considerando o ambiente excelente, com alta expectativa para o sucesso.

\section{REFERÊNCIAS}

1. Rennenberg MR, Gonçalves BS, Gonçalves MM. Design, design da informação e design instrucional: uma discussão necessária à evolução de um curso a distância. Design, Arte e Tecnologia 4. São Paulo: Rosari, Universidade Anhembi Morumbi, PUC-Rio e Unesp-Bauru,2008. 
2. Lacerda AL, Da Silva T. Possibilidades pedagógicas na perspectiva de uma educação online. Caderno Brasileiro de Ensino de Física. 2014;32(1):157-79.

3. Filatro A. Design instrucional contextualizado: educação e tecnologia. São Paulo: Pearson; 2007.

4. Filatro A. Design instrucional na prática. São Paulo: Pearson; 2008.

5. Tomazini EAS, Tobase L, Teodoro SV, Peres HHC, Almeida DM, Alavarce DC. Curso on-line sobre suporte avançado de vida em parada cardiorrespiratória: inovação para educação permanente. Rev Rene. 2018;19:e32444.

6. Marson LMD, Maximino LP, Domiciano CLC. Projeto didático digital para ensino de libras e português à crianças surdas, implantadas e ouvintes. In: Anais do XIX Jornada Fonoaudiológica de Bauru Profa. Dra. Katia Flores Genaro; Bauru, Brasil, p. 44-5, 2012.

7. Wellman B, Salaff J, Dimitrova D, Garton L, Gulia M, Haythornthwaite C. Computer networks as social networks: Collaborative work, telework, and virtual community. Annual Rev Sociol. 1996;22:213-38.

8. Silva AC, Silva CMT. Do diagnóstico às questões avaliativas: um caminho possível via prática de avaliação em educação a distância. Ensaio aval pol públ Educ. 2009;17(63): 293-312.

9. Aguiar ACL, Guimarães JMX, Ferreira HS, Almeida KTC, Ribeiro TFS, Anchieta TM et al. Blog como ferramenta educacional: contribuições para o processo interdisciplinar de educação em saúde. Rev Eletron Comun Inf Inov Saúde. 2018;12(2):220-31.

10. Struchiner $M$, Ramos $P$, Serpa Júnior $O D$. Desenvolvimento e implementação de um ambiente virtual de aprendizagem na área da saúde: uma experiência de pesquisa baseada em design. Interface. 2016;20(57):485-95.

11. Karnell MP, Bailey $\mathrm{P}$, Johnson $\mathrm{L}$. et al. Facilitating communication among speech pathologists treating children with cleft palate. Cleft Palate Craniofac. 2005;42(6):585-88.

12. Martins A, Franco EC, Caldana ML. Elaboração e avaliação de um website sobre 0 desenvolvimento da linguagem infantil: portal dos bebês - desenvolvimento da linguagem. Rev CEFAC. 2015;17(1):159-68.

13. Martins A. Telessaúde: Ambiente Virtual de Aprendizagem em aquisição e desenvolvimento da linguagem infantil [dissertação]. Bauru: Faculdade de Odontologia de Bauru, Universidade de São Paulo; 2013.

14. Small RV, Arnone MP. Motivation mining: prospecting the web. Book Report. 1999;18: 42-4.
15. Oliveira Júnior JF, Tubelo RA, Gradella G, Padilha JM, Zanatta EJ, Portella FF et al. O desigEJ, $\mathrm{n}$ da informação aplicado ao desenvolvimento da interface gráfica de um jogo sério sobre o combate ao mosquito Aedes aegypti. J Bras Tele. 2016;4(2):246-54.

16. Aguiar RV, Cassiani SHB. Desenvolvimento e avaliação de ambiente virtual de aprendizagem em curso profissionalizante de enfermagem. Rev Latino-Am Enferm. 2007;15(6):1086-91.

17. Brambilla CR, Dalpiaz GG, Silva AMM, Silva Junior M, Giraffa LMM, Ferreto TC. Ambiente colaborativo para formação de pessoal em medicina nuclear. Radiol Bras. 2011;44(3): 177-82.

18.Zeppone SC, Monti JF, Martins JR, Callegari FVR. Relato de experiência da construção de uma proposta para avaliação da prática profissional de um curso orientado por competências. Rev Bras Educ Med. 2016;40(4):757-64.

\section{CONFLITO DE INTERESSES}

Os autores declaram não haver conflitos de interesse

\section{AUTOR PARA CORRESPONDÊNCIA}

\section{Luciana Paula Maximino}

Al. Octávio Pinheiro Brisola, 9-75

17012-901 Bauru - SP, Brasil

lumaximino@uol.com.br
Submetido em 10/02/2020 Aceito em 23/10/2020 